\title{
A Continuity Principle for the Bergman Kernel Function
}

\author{
By \\ Klas Diederich* and Takeo OHSAWA**
}

\section{§. Statement of the Results}

Let $D \subset C^{n}$ be a bounded domain with $C^{\infty}$-smooth pseudoconvex boundary, and let $p \in \partial D$ be any point. By a two-sided bumping family of $D$ at $p$ we mean a family of smoothly bounded pseudoconvex domains $\left\{D_{t}\right\}_{-1 \leq t \leq 1}$ satisfying the following properties:

1) $D_{0}=D$,

2) $D_{t_{1}} \subset D_{t_{2}}$ if $t_{1}<t_{2}$,

3) $\left\{\partial D_{t}\right\}_{-1 \leq t \leq 1}$ is a $C^{\infty}$-family of real hypersurfaces in $C^{n}$,

4) for any neighborhood $U$ of $p$ in $C^{n}$ there exists a $t_{0}<0$ such that $D_{t_{0}} \backslash D_{-t_{0}} \subset U$.

Remark. A two-sided bumping family of $D$ at $p$ exists, of course, if $\partial D$ is strictly pseudoconvex at $p$. Recently, it was shown by Cho [Ch], that such a family also exists, if $\partial D$ is of finite type at $p$.

By a peak function at $p$ we mean a continuous function $f$ on $\bar{D}$ which is holomorphic on $D$ and satisfies $f(p)=1$ and $|f(z)|<1$ on $\bar{D} \backslash\{p\} . \quad$ By $K_{D}(z, w)$ we denote the Bergman kernel function of $D$ and we put $K_{D}(z)=K_{D}(z, z)$. Finally, we write $d s_{D}^{2}$ for the Bergman metric of $D$.

Our goal is to prove continuity results in the parameter $t$ of a bumping family for the Bergman kernel, the Bergman metric and related functions. Namely, we will show the following theorems:

Communicated by K. Saito, July 22, 1991.

1991 Mathematics Subject Classifications: 32 H 10

* Fachbereich 7 Mathematik, Bergische Universität GHS Wuppertal, Gauss Str. 20, D-5600 Wuppertal 1, Germany.

** Department of Mathematics, Nagoya University, 464-01 Nagoya, Japan. 
Theorem 1. Let $D \subset C^{n}$ be a bounded domain with $C^{\infty}$-smooth pseudoconvex. boundary and let $\left\{D_{t}\right\}_{-1 \leq t \leq 1}$ be a two-sided bumping family of $D$ at some point $p \in \partial D$ where $\partial D$ is strictly pseudoconvex. Then there is for any $\varepsilon>0$ and any neighborhood $U$ of $p$ a number $t_{0} \in(0,1)$ such that

$$
\left|K_{D}(z) K_{D_{t}}^{-1}(z)-1\right|<\varepsilon
$$

for all $t \in\left(-t_{0}, t_{0}\right)$ and $z \in D \backslash U$.

This theorem and the analogous result for the Bergman metric will be consequences of the continuity principle for so-called maximizing functions. We define:

Defimition 1. Let $D$ be a bounded domain in $\mathbb{C}^{n}$ and $w \in D$ an arbitrary point. We denote $\mathbb{N}_{0}=\mathbb{N} \cup\{0\}$, fix an $\alpha \in \mathbb{N}_{0}^{n}$ and put

$$
H_{(\alpha)}^{2}(D, w)=\left\{g \in H^{2}(D):\left(\frac{\partial}{\partial z}\right)^{\beta} g(w)=0 \forall \beta \in \mathbb{N}_{0}^{n} \text { with }|\beta| \leq|\alpha|, \beta \neq \alpha\right\} .
$$

By $B_{D}^{(\alpha)}(z, w) \in H_{(\alpha)}^{2}(D, w)$ we denote the (unique) function satisfying

$$
B_{D}^{(\alpha)}(w, w)=\max \left\{\left|\left(\frac{\partial}{\partial z}\right)^{\alpha} g(w)\right| \text { for } g \in H_{(\alpha)}^{2}(D, w) \text { with }\|g\|_{D}=1\right\}
$$

and we write $B_{D}^{(\alpha)}(w)=B_{D}^{(\alpha)}(w, w)$.

We will show

Theorem 2. Let $D, p \in \partial D$ be as in Theorem 1 and fix $\alpha \in \mathbb{N}_{0}^{n}$. Then there exists for any $\varepsilon>0$ and any neighborhood $U$ of $p$ a number $t_{\alpha} \in(0,1)$ such that

$$
\left|\frac{B_{D}^{(\alpha)}(w)}{B_{D_{t}}^{(\alpha)}(w)}-1\right|<\varepsilon
$$

for all $t \in\left(-t_{\alpha}, t_{\alpha}\right)$ and $w \in D \backslash U$.

As an immediate consequence one obtains

Corollay. Under the assumptions of Theorem 1 there exists for any $\varepsilon>0$ and any neighborhood $U$ of $p$ a $t^{\prime} \in(0,1)$ such that

$$
(1-\varepsilon) d s_{D}^{2} \leq d s_{D_{t}}^{2} \leq(1+\varepsilon) d s_{D}^{2}
$$

on $D \backslash U$ for all $t \in\left(-t^{\prime}, t^{\prime}\right)$.

\section{$\S 1$. The Maximizing Functions}

From now on, unless explicitly stated otherwise, we always suppose, that $D$ 
and $p \in \partial D$ satisfy the hypothesis of Theorem 1. Furthermore, we fix an $\alpha \in \boldsymbol{N}_{0}^{n}$ and let $B_{z}(w)=B(w, z)=B_{D}^{(\alpha)}(w, z)$ be the corresponding maximizing function as defined in Section 0. The first crucial tool for the proof of the theorems is the following

Lemma 1. For any $\varepsilon>0$ and any neighborhood $U$ of $p$ there exists $a t_{1} \in$ $(-1,0)$ such that

$$
\left\|B_{z}\right\|_{D \backslash D_{t}}^{2}<\varepsilon
$$

for all $t$ such that $t_{1}<t<0$ and for all $z \in D \backslash U$.

Proof. We may assume $\varepsilon<1$. Let $f$ be a peak function of $D$ at $p$. Since $\sup _{D \backslash U}|f|<1$ there exists an integer $m$ such that $\sup _{D \backslash U}|f|^{m}<\varepsilon / 4$. Therefore one can find a $t_{1} \in(-1,0)$ such that

$$
\sup _{D \backslash D_{t}}\left|1-f^{m}\right|<\frac{\varepsilon}{4}
$$

for all $t$ such that $t_{1}<t<0$.

Let $z \in D \backslash U$ be any point and put

$$
\psi_{z}=\left(1-f^{m}(z)\right)^{-1}\left(1-f^{m}\right) B_{z} .
$$

Then we have

$$
\left(\frac{\partial}{\partial w}\right)^{\beta} \psi_{z}(z)=\left(\frac{\partial}{\partial w}\right)^{\beta} B_{z}(z),
$$

for all $\beta \in \boldsymbol{N}_{0}^{n}$ with $|\beta| \leq|\alpha|$. Therefore $\left\|\psi_{z}\right\|_{D} \geq 1$.

On the other hand, one has

$$
\begin{aligned}
\left\|\psi_{z}\right\|_{D}^{2} & =\left(1-\frac{\varepsilon}{4}\right)^{-2}\left\|\left(1-f^{m}\right) B_{z}\right\|_{D}^{2} \\
& <\left(1-\frac{\varepsilon}{4}\right)^{-2}\left\|B_{z}\right\|_{D_{t}}^{2}+\frac{\varepsilon^{2}}{16}\left(1-\frac{\varepsilon}{4}\right)^{-2}\left\|B_{z}\right\|_{D \backslash D_{t}}^{2} \\
& =\left(1-\frac{\varepsilon}{4}\right)^{-2}-\left(1-\frac{\varepsilon^{2}}{16}\right)\left(1-\frac{\varepsilon}{4}\right)^{-2}\left\|B_{z}\right\|_{D \backslash D_{t}}^{2} .
\end{aligned}
$$

Hence we get

$$
\left\|B_{z}\right\|_{D \backslash D_{t}}^{2}<\left\{1-\left(1-\frac{\varepsilon}{4}\right)^{2}\right\}\left(1-\frac{\varepsilon^{2}}{16}\right)^{-1}<\varepsilon .
$$

This finishes the proof. 


\section{§2. An Approximation Lemma}

The second technical lemma needed for the proof of the theorems deals with uniform approximation on bumping families. For this we fix such a family $\left\{D_{t}\right\}_{-1 \leq t \leq 1}$ of $D$ at $p$. We can choose a corresponding $C^{\infty}$-family $\left\{\rho_{t}\right\}$ of defining functions, i.e. a $C^{\infty}$ function $\rho_{t}(z)=\rho(t, z)$ on $[-1,1] \times \mathbb{C}^{n}$ such that $D_{t}=$ $\left\{z \in \mathbb{C}^{n}: \rho_{t}(z)<0\right\}$ and $d \rho_{t} \neq 0$ on $\partial D_{t}$ and such that the functions $\rho_{t}(z)$ are strictly plurisubharmonic in a neighborhood of $\overline{D_{1} \backslash D_{-1}}$. With this we put $r_{t}=2 \rho_{0}$ $-\rho_{t}$ and $U_{t}=\left\{z \in D: r_{t}(z)<0\right\}$.

We show:

Lemma. There exists a constant $C$ such that for any $\varphi \in H^{2}(D)$ and $t \in[0,1]$ one can find $\varphi_{t} \in H^{2}\left(D_{t}\right)$ satisfying

$$
\left\|\varphi-\varphi_{t}\right\|_{D} \leq C\|\varphi\|_{U_{t}}
$$

and

$$
\left\|\varphi_{t}\right\|_{D_{t}} \leq\|\varphi\|_{D}+C\|\varphi\|_{U_{t}} .
$$

Proof. We put $h_{t}=\rho_{t}^{-1} r_{t}$. Then

$$
\begin{aligned}
\partial h_{t} & =-h_{t} \rho_{t}^{-1} \partial \rho_{t}+\rho_{t}^{-1} \partial r_{t} \\
& =-\left(h_{t}+1\right) \rho_{t}^{-1} \partial \rho_{t}+2 \rho_{t}^{-1} \partial \rho_{0} .
\end{aligned}
$$

Note that there exist constants $C_{0}$ and $C_{\varepsilon}$ (for any $\varepsilon>0$ ) such that

$$
\partial \rho_{0} \bar{\partial} \rho_{0} \leq C_{0} \partial \rho_{t} \bar{\partial} \rho_{t}+C_{\varepsilon}\left|\rho_{t}\right|^{2-\varepsilon} d s_{e}^{2} \text { on } D
$$

where $d s_{e}^{2}$ denotes the eulidean metric.

Let $d s_{t}^{2}$ be the metric on $D_{t}$ defined by

$$
d s_{\S}^{2}=d s_{e}^{2}+c_{0} \partial \bar{\partial} \log \left(-\rho_{t}\right),
$$

where $\partial \bar{\partial} \log \left(-\rho_{t}\right)$ stands for the complex Hessian of $\log \left(-\rho_{t}\right)$, and $c_{0}$ is a sufficiently small positive constant, so that $d s_{t}^{2}$ is a metric for all $t$. Let $|\cdot|_{t}$ denote the pointwise length with respect to $d s_{t}^{2}$. Then, combining the strict plurisubharmonicity of $\rho_{t}$ with (1) and (2), we obtain an estimate

$$
\left|\partial h_{t}\right|_{t}^{2} \leq-2 c_{0}\left(h_{t}+1\right)^{2}+C_{\varepsilon}^{\prime}\left|\rho_{0}\right|^{2-\varepsilon} \rho_{t}^{-1} \text { on } D
$$

for all $\varepsilon>0$. Here $C_{\varepsilon}^{\prime}$ may depend on $\varepsilon$.

Note that $U_{t}=\left\{z \in D_{t}:-1<h_{t}(z)<0\right\}$. Hence 


$$
\left|\rho_{t}\right|=\left|h_{t}^{-1} r_{t}\right| \geq\left|r_{t}\right| \geq 2\left|\rho_{0}\right|-\left|\rho_{t}\right| \text { on } U_{t} .
$$

Therefore, one has on $U_{t}$

$$
\left|\partial h_{t}\right|_{t}^{2} \leq-2 c_{0}+C_{\varepsilon}^{\prime}\left|\rho_{0}\right|^{1-\varepsilon}
$$

In particular, we obtain on $U_{t}$ for some constant $C_{1}$

$$
\left|\partial h_{t}\right|_{t}^{2} \leq C_{1} .
$$

Let $\chi: \boldsymbol{R} \rightarrow[0,1]$ be any $\mathrm{C}^{\infty}$-function satisfying $\chi=0$ on $(-\infty,-1]$ and $\chi=1$ on $[0, \infty)$. Given any function $\varphi \in H^{2}(D)$ and $t \in[0,1]$ we put

$$
u_{t}=\bar{\partial}\left(\chi\left(h_{t}\right) \varphi\right) \wedge d z_{1} \wedge \cdots \wedge d z_{n} .
$$

By (4) there exists a constant $C_{2}$ independent of $t$ such that

$$
\left\|u_{t}\right\|_{t} \leq C_{2}\|\varphi\|_{U_{t}}
$$

where $\|\cdot\|_{t}$ denotes the $L^{2}$-norm with respect to $d s_{t}^{2}$. Since the potential function $\|z\|^{2}-c_{0} \log \left(-\rho_{t}\right)$ of $d s_{t}^{2}$ has a bounded gradient with respect to $d s_{t}^{2}$, with the bound $C_{3}$ being independent of $t$, in virtue of the $L^{2}$-vanishing theorem of Donnelly-Fefferman [D-F] there exists an $(n, 0)$-form $v_{t}$ on $D_{t}$ satisfying

$$
\bar{\partial} v_{t}=u_{t} \text { and }\left\|v_{t}\right\|_{D_{t}} \leq C_{3}\left\|u_{t}\right\|_{t},
$$

where we note that the $L^{2}$-norm of $v_{t}$ does not depend on the choice of Hermitian metrics since $v_{t}$ is of type $(\mathrm{n}, 0)$. Let us now define $\varphi_{t} \in H^{2}\left(D_{t}\right)$ by

$$
\varphi_{t} d z_{1} \wedge \cdots \wedge d z_{n}=\chi\left(h_{t}\right) \varphi \wedge d z_{1} \wedge \cdots \wedge d z_{n}-v_{t}
$$

Then we obtain

$$
\left\|\varphi-\varphi_{t}\right\|_{D} \leq\left(1+C_{2} C_{3}\right)\|\varphi\|_{U_{t}}
$$

and

$$
\left\|\varphi_{t}\right\|_{D_{t}} \leq\|\varphi\|_{D}+C_{2} C_{3}\|\varphi\|_{U_{t}} .
$$

Thus the constant $C=1+C_{2} C_{3}$ satisfies the requirement.

\section{§3. The Proof of the Continuity Principle}

In order to avoid hiding the essentials behind technical details, we will prove here in detail Theorem 2 for the case $\alpha=0$. Theorem 1 is an immediate consequence of this. At the end we will then indicate, how the proof has to be modified in order to give Theorem 2 for general $\alpha$. 
Let $D,\left\{D_{t}\right\}_{-1 \leq t \leq 1}, p \in \partial D$ and the neighborhood $U$ of $p$ be given so as to satisfy the conditions of Theorem 1 , take $z \in D \backslash U$ and let $B_{z}=B_{D}^{(0)}\left({ }^{\circ}, z\right)$ be the corresponding maximizing function. Choose a smooth family of defining functions $\rho_{t}$ for the bumping family as in Section 2. By applying the approximation lemma we obtain functions $B_{z, t} \in H^{2}\left(D_{t}\right)$ for $0<t \ll 1$ such that

$$
\left\|B_{z, t}-B_{z}\right\|_{D}^{2} \leq C\left\|B_{z}\right\|_{U_{t}}^{2} \text { and }\left\|B_{z, t}\right\|_{D}^{2} \leq 1+C\left\|B_{z}\right\|_{U_{t}}^{2} \text {. }
$$

Here $U_{t}$ is as before and $C$ is a constant independent of $t$. Hence we obtain

$$
\left|B_{z, t}(z)-B_{z}(z)\right|^{2} \leq C\left\|B_{z}\right\|_{U_{t}}^{2} K_{D}(z)
$$

and

$$
K_{D_{t}}(z) \geq\left(1+C\left\|B_{z}\right\|_{U_{t}}^{2}\right)^{-1}\left|B_{z, t}(z)\right|^{2} .
$$

Combining (5) and (6) we obtain

$$
K_{D_{t}}(z) \geq\left(1+C\left\|B_{z}\right\|_{U_{t}}^{2}\right)^{-1}\left(1-C\left\|B_{z}\right\|_{U_{t}}^{2}\right) K_{D}(z) .
$$

Since $K_{D_{t}}(z) \leq K_{D}(z)$ we have

$$
\lim _{t \rightarrow+0} K_{D}(z) K_{D_{t}}^{-1}(z)=1
$$

by Lemma 1 . On the other hand it follows directly from Lemma 1 , that

$$
\lim _{t \rightarrow-0} K_{D}(z) K_{D_{t}}^{-1}(z)=1
$$

Thus the proof of Theorem 2 for $\alpha=0$ and of Theorem 1 is finished.

The proof of Theorem 2 for arbitrary $\alpha$ is completely similar except that we must have approximating functions $B_{z, t}^{(\alpha)} \in H_{(\alpha)}^{2}\left(D_{t}, z\right)$ for $B_{D}^{(\alpha)}(\cdot, z)$. In order to realize this additional restriction one only has to replace the use of DonnellyFefferman's vanishing theorem by that with weight functions of the form $N$ $\log |z-w|^{2}(N \gg 1)$. For such a modification of Donnelly-Fefferman's vanishing theorem the reader is referred to Ohsawa-Takegoshi [O-T].

In order to see, that the Corollary on the Bergman metric follows from Theorem 2, we only have to recall, that

$$
d s_{D}^{2}(X, X)=\sup \left\{|X g(z)|^{2} K_{D}^{-1}(z):\|g\|_{D}=1 \text { and } g(z)=0\right\}
$$

for any $z \in D$ and any tangent vector $X$ at $z$.

\section{References}

[Ch] Cho, S., Extension of complex structures on weakly pseudoconvex compact complex 
manifolds with boundary, Preprint 1991.

[D-F] Donnelly, H. and Fefferman, Ch., $L^{2}$-cohomology and index theorem for the Bergman metric, Ann. Math., 118 (1984), 593-619.

[O-T] Ohsawa, T. and Takegoshi, K., Hodge spectral sequence on pseudoconvex domains, Math. Z., 197 (1988), 1-12. 
